\title{
Bücherschau.
}

Oeuvres Complètes de J.-C. Galissard de Marignac. Tome II. Mémoires et Critiques 1860-1887. Hors-Série des Mémoires publiés sous les auspices de la Société de Physique et d'Histoire Naturelle de Genève par E. ADor. 1903.

Vor einiger Zeit haben wir in dieser Zeitschrift (Bd. 33, S. 390-92) ein Referat über den ersten Band der gesammelten Werke Marignacs gebracht. In dem vor uns liegenden zweiten Bande begegnen wir dem Genius der anorganischen Chemie auf der Höhe seiner wissenschaftlichen Tätigkeit. Wir finden hier wiederum Arbeiten aus allen Gebieten der Chemie, mit Ausschlufs der organisehen Chemie. Es ist unmöglich, selbst nur die blolsen Titel der 62 Originalabhandlungen und kritischen Referate über hervorragende Arbeiten der Zeitgenossen anzuführen, und wir müssen uns nur auf die Anführung der bedeutendsten Leistungen beschränken.

An erster Stelle sei die Arbeit über die Fluorzirkonate erwähnt, durch welche die Formel $\mathrm{ZrO}_{2}$ für das Oxyd und das Atomgewicht $\mathrm{Zr}=90$ für das Element definitiv festgestellt worden sind. Ferner folgen seine bekannte geistreiche Methode der Bestimmung der Borsäure, sowie die grundlegenden Untersuchungen der Wolframate, Fluorwolframate und Silicowolframate. Aber das grofsartigste Monument des wissenschaftlichen Ruhmes hat sich MaRrgNac durch seine klassischen Arbeiten über das Niob und Tantal gestellt. HernRICH Rose, ein Chemiker ersten Ranges, bemühte sich fast sein halbes Leben lang, um eine klare Idee über das Tantal und Niob und ihre Verbindungen zu erhalten, und doch gelangte er nicht zu einem richtigen Resultat. Nach ihm bildet das Niob $(\mathrm{Nb}=97.6)$ zwei Oxyde, $\mathrm{Nb}_{2} \mathrm{O}_{3}$ und $\mathrm{NbO}_{2}$, und das Oxyd des Tantals ( $\mathrm{Ta}=137.6$ ) hielt er für $\mathrm{TaO}_{2}$. Marignac erkannte, dafs die dunkeln, diesen Gegenstand betreffenden Fragen durch das bisherige Studium der blofsen Sauerstoffverbindungen nicht gelöst werden können, und widmete deshalb seine Aufmerksamkeit den Fluoriden, wie er dies schon früher mit grofsem Erfolg bei den Elementen Si, Ti, Zr, Sn getan hatte. Er fand, dals 
unter den gleichen Bedingungen das Niob eine gröfsere Neigung zur Bildung der Doppelsalze des Oxyfluorids $\mathrm{NbOF}_{3}$, das Tantal aber solcher des Fluorids $\mathrm{TaF}_{5}$ besitzt, da es stärker basisch ist. Auf Grund der verschiedenen Löslichkeit der betreffenden Doppelfluoride berei亡ete Marignac zum erstenmal reine Verbindungen sowohl des Niobs, wie auch des Tantals. Aus diesen Arbeiten folgt zunächst der Isomorphismus des Sauerstoffs mit dem Fluor und ferner der für die spätere Erbauung des periodischen Systems theoretisch wichtige Befund, dals sich das Niob $(\mathrm{Nb}=94)$ zum Molybdän $($ Mo $=96)$ so verhält, wie das Tantal (Ta $=182)$ zum Wolfram ( $W=184)$. Wenn wir diese Atomgewichte und Beziehungen mit dem vergleichen, was der unmittelbare Vorgänger des periodischen Systems, nämlich ,The law of octaves" von NwwLands enthielt, in welchem wir eine so wenig harmonische Aufeinanderfolge der "Töne* wie: $\mathrm{Ba}=137, \mathrm{~V}=137$ (!) $\mathrm{Ta}=138$ (!), $\mathrm{W}=184, \mathrm{Nb}=195$ (!) finden, so ersehen wir, dals erst die zitierten Arbeiten Marignacs, zusammen mit den klassischen Untersuchungen Roscoes, die Aufstellung unserer jetzigen fünften Gruppe im periodischen System ermöglichten, ebenso wie Marignacs frühere Untersuchungen über die Elemente Si, Ti, $\mathrm{Zr}$ und Sn das Hauptmaterial zur Aufstellung der vierten Gruppe geliefert haben. Die Elemente Mo und $W$ bilden dann die Fortsetzung der Reihen $\mathrm{Zr}$, $\mathrm{Nb}$ und $\mathrm{Ta}$ in der sechsten Gruppe. Ein weiteres wichtiges Glied dieser Reihe von Arbeiten bildet die Trennung des Niobs von Titan und der Nachweis der Nichtexistenz des Ilmeniums.

Im Fluge erwähne ich nur die die latente Verdampfungswärme des Salmiaks und anderer Stoffe betreffende Untersuchung. MarrGNac fand, dal's die latente Verdampfungswärme des Salmiaks und die zu seiner Zersetzung nötige Wärme fast gleich sind, woraus ein für die damalige Zeit (1868) hochwichtiger Schlufs folgt, dals der Salmiak durch Erhitzen in seine gasförmigen Bestandteile dissoziiert wird. Ganz ähnliche Verhältnisse beobachtete Marignac beim Übergang der Schwefelsäure in den Dampfzustand, wobei eine Spaltung in das Anhydrid und Wasserdampf eintritt.

Von fundamentaler Bedeutung sind Marignacs Arbeiten über wässerige Lösungen $(1869,1870)$. In diesen Arbeiten verfolgte er die die Verdünnung begleitenden Erscheinungen und fand, dafs gewisse Anomalien, welche eintreten, je nachdem man zwei Lösungen entweder zuerst mischt und dann verdünnt, oder ungekehrt verfährt, durch die Veränderung der

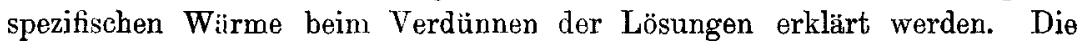
Verdünnung ist stets von einer Kontraktion begleitet und die wässerige Lösung besitzt stets einen grölseren Ausdebnungskoëffizienten, als dem Durchschnitt der Koëffizienten der beiden gemischten Lösungen entspricht. Bei sehr hoher Temperatur würde die Verdünnung ohne Kontraktion und Verminderung der spezifischen Wärme stattfinden. Im Jahre 1876 be- 
schäftigte sich Marianac mit der Lösung der Frage, ob es nicht möglich wäre, die spezifischen Wärmen der Lösungen für verschiedene Salze in der Art zu berechnen, wie dies für die Dichte mit Hilfe der VaLsosschen Module möglich ist, es wurden aber kompliziertere Verhältnisse konstatiert, welche erst durch unsere moderne Theorie der Lösungen ihre Erklärung finden.

Weiter seien Marignacs Arbeiten über die Löslichkeit des Calciumsulfats in Wasser und die dabei beobschteten Übersättigungserscheinungen (1873) angeführt, ferner seine Arbeit über die gleichzeitige Diffusion mehrerer Salze, aus welcher hervorgeht, dafs das Gemisch zweier Substanzen stets die Diffusibilität derjenigen der beiden Substanzen vermindert, welche schwieriger diffundiert. Interessant ist besonders der Befund, dals sowohl die Salze bestimmter Süuren stets die gleiche, von dem Charakter der Säure unabhängige Ordnung der Diffusionsgeschwindigkeit, als auch, dals alle Salze von irgend welchem Metall eine von dem Charakter der Säure unabhängige Ordnung bewahren. Wir wissen jetzt, dafs die Ordnung der Diffusionskoëffizienten verschiedener Anionen und Kationen in der Leitfähigkeit der Lösungen ihre Analogie findet.

Reminiszenzen eigentümlicher Art, verbunden mit Gefühlen der Verwunderung darüber, wie konservativ gewisse Kreise auch in den exakten Wissenschaften sein können, werden durch Martenacs Abhandlung aus dem Sahre 1877 über die Äquivalente und Atomgewichte als Grundlagen des Systems der chemischen Notation geweckt. Im Jahre 1858 haben Marignac und Cannizzaro die ausschlielsliche Berechtigung der atomistischen Notation, welche zu den grolsartigen Ideen Karulés über die Struktur der organischen Verbindungen führte und giänzende Resultate auf dem Gebiete der chemischen Synthese ermöglichte, nachgewiesen. Nicht weniger glänzend war die Anwendung der neuen Notation auf dem Gebiete der anorganischen Chemie. Im Jahre 1869 und 1870 hatte MenDELEJEFF gezeigt, dals die Anordnung der Elemente auf Grund ihrer zanehmenden Atomgewichte, nicht aber Äquivalente, zu der grolsartigen Generalisation führt, welche wir als das periodische System und Gesetz bezeichnen. Dies ermöglichte MeNDELejefr, vorauszusagen, dafs „der Begriff vom Atomgewicht.... unter allem Wechsel in den theoretischen Vorstellungen der Chemiker sich obne Änderung erhalten wird." Und nun, bedenken wir, widmet die Pariser Akademie im Jahre 1877 mehrere Sitzungen der Diskussion darüber, ob die Notation nach den Äquivalenten oder nach den Atomgewichten berechtigt sei. Dabei wird, zwei Jahre nach der Entdeckung des Galliums, wodurch die Richtigkeit des auf Atomgewichte begründeten periodischen Systems glünzend dargetan wurde, die Berechtigung der atomistischen Notation von den gröfsten Koryphäen der Wissenschaft geleugnet! Marigasac gelangte in eine Polemik mit BERTHeLot und weist dem letzteren durch gewichtige logische 
Argumente die alleinige Berechtigung des atomistischen Standpunktes nach, also des Standpunktes, auf welchem heute alle Chemiker und auch Herr Berthelot stehen.

Wir gelangen nun zu einem Gebiete, auf welchem der Name MARIGNACs als derjenige eines der hervorragendsten Arbeiter stets genannt werden wird, zur Chemie der seltenen Erden. Ich erwähne nur kurz die Hauptresultate seiner Arbeiten. Die erste betrifft die Bestimmung der Atomgewichte des Cers, Lanthaus und Didyms (1848). Bei derartigen Arbeiten liegen die Schwierigkeiten nicht nur in der Bestimmung der Atomgewichte, sondern auch in der Darstellung des reinen Materials, und wenn wir bedenken, dafs die vorliegende Arbeit in die ersten Jahre der Entwickelung dieses Teiles unserer Wissenschaft fällt, welche eigentlich erst durch die genialen Arbeiten Mosanders (seit 1839) inauguriert wurde, so erkennen wir, welche Schwierigkeiten MarigNac zu überwinden hatte, als er sich auf diesem Felde erst die Bahn zu brechen begann. Es folgen dann eine ausführliche Untersuchung über das Didym. (1853) und mehrere Arbeiten über die Krystallformen der Salze der seltenen Erden (1855).

Nach langer, besonders dem Studium der Verbindungen des Zirkoniums, des Wolframs, Molybdäns, Tantals und Niobs gewidmeter Periode kehrt Marignac erst im Jahre $1873 \mathrm{zu}$ den seltenen Erden zurück. In einer Abhandlung über die Salze des Berylliums und der Ceritelemente beschreibt Marignac die Alkalidoppelfluoride des Berylliums und behandelt gleichzeitig die Frage, ob dieses Element als zweiwertig oder dreiwertig anzusehen sei. Es wird eine Anzahl von Argumenten pro und contra diskutiert. Die Zusammensetzung der Doppelfluoride scheint ihm für die Zweiwertigkeit des Berylliums zu sprechen, er findet aber, dals sein Sulfat weder mit dem Sulfat des Aluminiums, noch mit den Sulfaten der zweiwertigen Elemente zusammen krystallisiert. Auch das Nitrat ist nicht isomorph mit den Nitraten von $\mathrm{Mg}, \mathrm{La}, \mathrm{Di}$, welche letatere damals noch für zweiwertig gehalten wurden. Neben der Beschreibung und Messung der Krystalle einiger Salze seltener Erden finden wir in dieser Abhandlung auch einige neue, das Atomgewicht des Lanthans betreffende Daten.

Marignac hielt die seltenen Erden, trotz der Argumente MendeisJEFPS aus dem Jahre 1870, für RO, und erst auf Grund der Arbeiten Curves aus dem Jahre 1874 schlols er sich der Ansicht an, nach welcher diese Erden die Formel $\mathrm{R}_{\mathbf{2}} \mathrm{O}_{3}$ besitzen.

In seiner grolsen Arbeit über die Gadoliniterden (1878) widmet sich Marigrac dem Studium des Terbiums, dessen Atomgewicht er zu 148.5 findet, und zugleich wird die Existenz einer neuen Erde angekündigt, an deren spektralanalytischer Entdeckung SORET teilgenommen hat. Es ist die Erde X oder das später entdeckte Holmiumoxyd von CLeve. In einer weiteren Notiz beweist Manignac, dals das Mosandrum von L. Sмiтн 
hauptsæichlich aus Terbium besteht, aber daneben kleine Mengen anderer Erden enthält.

Im Jahre 1878 hat Marignac die Bunswa-Barrsche Methode der Fraktionierung der Yttriumerden durch sukzessive Zersetgung der Nitrate beim Erhitzen, eine wahre Differenzierung der seltenen Erden, in der Weise modifiziert, dafs er die Nitrate auf eine bedeutend höhere Temperatur erhitzte, als dies BuNSEN tat, nämlich bis die geschmolzenen Nitrate infolge der Zersetzung dickflüssig wurden oder sogar erstarrten. Marignad wies nach, dafs das bisherige Erbium sich auf diese Weise zerlegen läfst in das wahre, rosenrote Salze liefernde Erbium und in ein neues, farblose Salze lieferndes Erdelement, welches er als Ytterbium, $\mathrm{Yb}=172.5$, bezeichnete. Es ist. bekannt, dafs es durch Fortsetzung dieses Verfahrens mit grölseren Mengen Material in den Jahren 1879 und 1880 Nusson gelungen ist, das Ytterbium Marignacs in zwei Elemente $\mathrm{zu}$ zerlegen: in das wahre Ytterbium mit dem Atomgewicht 173 und in das neue Element Scandium, $\mathrm{Se}=44$, welehes mit dem von MendedejefF vorausgesagten Ekabor identisch ist.

Im Jahre 1880 erschien eine weitere fundamentale Arbeit Marignacs: Über die Erden des Samarskits. Er verwandte diesmal zur Trennung der einzelnen Erden neben der Nitratmethode ein neues, auf der verschieden grolsen Löslichkeit der Kaliumdoppelsulfate der Erden begründetes Fraktionierungsverfahren. Durch vielfache Wiederholung dieses Prozesses mit Fraktionen von verschiedener Löslichkeit gelang es Marignac, zwei neue Erden zo entdecken. Die eine bezeichnet er als $\mathrm{Y} \alpha$, die andere als $\mathrm{Y} \beta$. Das Atomgewicht des Elementes aus $\mathrm{Y} \alpha$ fand er $=156.75$. Dieses Element wurde im Jahre 1886 als Gadolinium bezeichnet und durch die später von Lecoq, CLeVE, BetrendorfF und BenenIKs ausgeführten Untersuchungen wurde sowohl seine Existenz, als auch sein Atomgewicht, welches rund $G d=156$ beträgt, bestätigt. Die andere Erde, Y $\beta$, erwies sich als identisch mit dem Hauptbestandteil des Decipiums von DELAfoNTAINe und mit dem 1879 von LecoQ entdeckten Samarium. MarignaC fand das Atomgewicht des Elements $Y \beta=149.4$, später fanden BraunER, Cleve, Bettendorff $\mathrm{Sm}=150$. Nach Demarģay soll im Samarium noch das farblose Salze liefernde Europinm enthalten sein $(\mathbf{E u}=151)$, so dafs das Atomgewicht des reinen Samariums der ursprünglichen Zahl Marigracs näher kommen dürfte.

Die letzte Arbeit Marignacs über seltene Erden datiert aus dem Jahre 1887. Es ist überhaupt die letzte Publikation dieses grofsen Forschers und bezieht sich auf die Crookessche Theorie der Genesis der Elemente. Vorher hat Marigsac noch (1884) eine grofse, auf mehrjährige Versuche begründete Arbeit über die Veränderlichkeit des Gebaltes an organischen Substanzen im Wasser der Rhône bei ihrem Ausflusse aus dem Genfer See veröffentlicht. 
Es erübrigt noch, die die Atomgewichtsbestimmungen des Wismuts, Mangans, Zinks und Magnesiums betreffenden Arbeiten Marianacs aus dem Jahre 1883 kurz zu erwühnen, welche zu den Zahlen $\mathrm{Bi}=208.2$ bis 208.6, $\mathrm{Mn}=55.07, \mathrm{Zn}=65.33$ und $\mathrm{Mg}=24.37$ geführt haben. Diese Zahlen, durch einfache Methoden erhalten, sind mit den heute angenommenen internationalen Atomgewichten fast identisch, und ähnliche kleine Abweichungen ergeben sich meistens auch in Bezug auf die von MARIGNAC ausgeführten Atomgewichtsbestimmungen von im ganzen 30 Elementen, wie eine Zusammenstellung auf S. 823 zeigt.

In der Einleitung za den genannten Arbeiten teilt Marignac eine Anzahl von wichtigen Gründen mit, welche ihn bewogen haben, die Atomgewichte nicht auf $\mathrm{H}=1$, sondern auf die unveränderliche Basis $\mathrm{O}=16$ zu beziehen. Da aber im Jahre 1883 diese Frage keine Tagesfrage der Chemie bildete, so wurde der Vorschlag Marignacs von den Chemikern damals nicht berücksichtigt. Der Vorschlag, der zum erstenmal von STAS im Jahre 1865 gemacht wurde, wurde im Jahre 1888 vom Referenten wiederholt und bildet erst seit dieser Zeit eine wichtige Tagesfrage der Chemie. In den Jahren 1889, 1897, 1901 und später wurde er vom Referenten und einer Anzahl anderer Chemiker durch neve Argumente dargelegt, so dal's heute die überwiegende Mehrzahl der anorganischen und physikalisehen Chemiker die auf $0=16$ bezogenen Atomgewichte benützt. Dagegen haben sich hauptsächlich die Organiker, welche sich im Gegensatz zu den Anorganikern eigentlich nur für eine beschränkte Anzahl von Elementen interessieren, für $\mathrm{H}=1$ ausgesprochen. In der Tat benützen sie aber, wie OsTwald richtig bemerkte, bei ihren Berechnungen die Zahlen $\mathrm{H}=1,0=16, \mathrm{C}=12, \mathrm{~N}=14$ n. s. w., was übrigens begreiflich ist, da bei den Fehlerquellen, denen die Methoden der Elementaranalyse bekanntlich unterliegen, die geringen Abweichungen der runden Zahlen von den richtigen Zahlen praktiseh bedeutungslos sind.

Ich habe vorliegend nur einen kurzen Überblick der intensiven wissenschaftlichen Tätigkeit und damit auch der Lebenstätigkeit unseres Klassikers der anorganischen und physikalischen Chemie, den ich als eineu meiner besten, obwohl nur indirekten Lehrer betrachte, gegeben, und heute, wo die beiden genannten Zwweige unserer Wissenschaft sich so vielversprechend, eine von der anderen unterstützt, entwickeln, und wo die Arbeiten Marignacs auf so leichte Art zugänglich gemacht worden sind (der Preis der beiden starken Bände beträgt nur $20 \mathrm{Mk}$.), ist es geradezu die Pflicht der modernen anorganischen und physikalisehen Chemiker, dem vor uns liegenden klassischen Werke ein gründliches Studium zu widmes.

\section{Bohuslav Brauner.}

J. H. van't Hoff. Die Phasenlehre. Vortrag, gehalten vor der Deutschen Chem. Gesellschaft. (Ber. deutsch. chem. Ges. 35, 4252-4265; 1902.) 